Journal of Machine Engineering, 2021, Vol. 21, No. 1, 121-132

ISSN 1895-7595 (Print) ISSN 2391-8071 (Online)

Received: 02 December 2020 / Accepted: 26 February 2021 / Published online: 29 March 2021

ISO GPS, geometrical tolerancing, aluminium extrusion, product verification

\author{
Zbigniew HUMIENNY ${ }^{1^{*}}$
}

Marcin BLASZCZYK ${ }^{2}$

\title{
TOLERANCES FOR ALUMINIUM ALLOY-EXTRUDED PROFILES ACCORDING TO EN 755-9 AND EN 12020-2 VERSUS DEFINITIONS OF TOLERANCES ACCORDING TO THE ISO GPS SYSTEM
}

\begin{abstract}
The inconsistencies between tolerances for aluminium profiles as specified in EN 755-9:2016 along with EN 12020-2:2016 and tolerance concepts defined in ISO 1101:2017 are demonstrated. It is shown that the standards defining requirements for extruded profiles have been developed without considering provisions in the ISO GPS system. A number of ambiguities in EN 755-9 and EN 12020-2 that were overcome in the ISO GPS system are shown. For example, broad application of plus/minus tolerancing for step dimensions does not provide unique verification of aluminium alloy-extruded profiles. It is pointed out that some terms univocally defined in the ISO GPS system are used incorrectly in both standards. It makes it difficult for a customer to verify on CMM whether or not extruded profiles satisfy the requirements specified in the order. Finally, significant changes regarding geometrical tolerancing in standards for the aluminium alloy-extruded profiles are proposed.
\end{abstract}

\section{INTRODUCTION}

The aluminium alloy-extruded profiles that are widely used in the automated storage and picking systems designed by Autostore [1] require a relevant geometrical accuracy of the utilized profiles. Unfortunately, European Standards defining requirements for extruded profiles have been developed without considering provisions in the ISO Geometrical Product Specification system (ISO GPS system) that has been adopted worldwide. The first fundamental difference between EN 755-9:2016 [2] along with EN 12020-2:2016 [3], and tolerance concepts defined in International Standards ISO 1101:2017 [4], and also in ISO 1660:2017 [5] is a wide application of $+/-$ tolerancing for extruded profiles for dimensions other than linear or angular size, that produces ambiguous requirements when applied to the real workpiece. The presence of form and angular deviations on all real workpieces make requirements specified in [3] ambiguous and therefore geometrical tolerances should be used to control the specification ambiguity [6]. This $+/-$ specification ambiguity can only be avoided for features of size (a cylinder, a sphere, two parallel opposite planes, a circle, two parallel opposite straight lines and two opposite circles) [7, 8].

\footnotetext{
${ }^{1}$ Institute of Machine Design Fundamentals, Warsaw University of Technology, Warsaw, Poland

${ }^{2}$ Research \& Development, AutoStore Sp. z o.o., Poland

*E-mail: zbigniew.humienny@pw.edu.pl https://doi.org./10.36897/jme/133728
} 
Therefore, that some of the dimensions indicated as " $\mathrm{H}$ " in Fig. 1 [3] are dimensions other than linear size the $+/-$ tolerancing cannot be applied without specification ambiguity, as will be explained in the further part of this text (Section 3). Applying dimension " $\mathrm{H}$ " to centre distances or linear step dimensions is in contradiction to the GPS rules [9]. Furthermore, several definitions of size are given in the ISO GPS system standards [7-9] while standard EN 12020 does not provide any terms for size. These terms are also required for the correct interpretation of the rules described in [10].

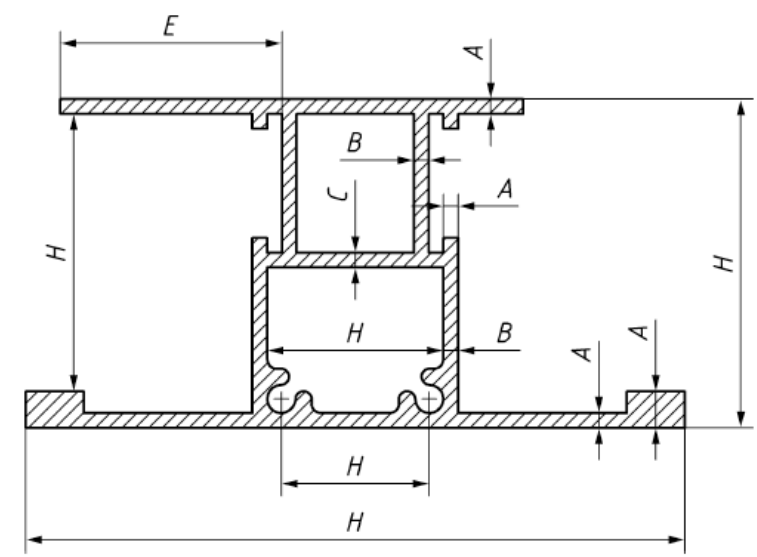

Fig. 1. Ambiguous definitions of dimensions for hollow extruded profile are given in [3]

The second fundamental difference is the lack of a proper workpiece datum system [11]. The tolerances of dimensions given in [3] are applied individually, i.e. independently from each other (without a datum system). It is difficult or even impossible to assess what the overall shape of the workpiece can be accepted. The description such as "longer planes shall be taken as contact reference" is used in EN 12020-2:2016 for the tolerances on angularity defined peculiarly instead of a clear definition of datum system. This can cause ambiguities during profile verification for equal-side lengths. The functional surface shall be clearly indicated. Corresponding interfacing features of mating parts should be selected as datum features and indicated to ensure proper assembly.

In the following text, only standards are given as references however recently published textbooks that present the ISO GPS system geometrical tolerancing rules and concepts applied in the paper shall be listed $[12,13]$. If need, the reader can refer to those textbooks to find more information on the ISO GPS system as well as to better understand details of proposed revisions of EN 755-9:2016 and EN 12020-2:2016.

\section{AMBIGUOUS TERMS AND DEFINITIONS}

The standard [3] lists [4] as a normative reference, but this standard also contains new terms (actually new wording) used for concepts well known in GPS. This applies to terms such as "theoretical exact line", "contour", "convexity - concavity", "twist" or "angularity". 
Those terms and their descriptions are closest to ISO GPS's "profile of a line", "flatness", "perpendicularity" or "position". These examples will be discussed in the following section along with other aspects which create ambiguities.

Taking under consideration [11], the parallelism requirement specified in Fig. 2 is in contradiction to verification routines also presented in this figure. According to datum specification, a datum "A" shall be an integral, nominally planar surface, however, a workpiece is placed on two supports while there is no formal call-out of datum targets. This may have a direct impact on deviation values due to the limited stiffness of a profile, so it may also have an impact on reproducibility results. Therefore, the example given in [3], does not correctly recognize an indication of the design intent. On the other hand, the description "two clearly defined points shall specify the width of the parallelism measurement" [5] may be interpreted as the limits for extension of the target line while datum feature indicator A according to [11] implies single datum, i.e. datum established from one datum feature taken from a single surface.

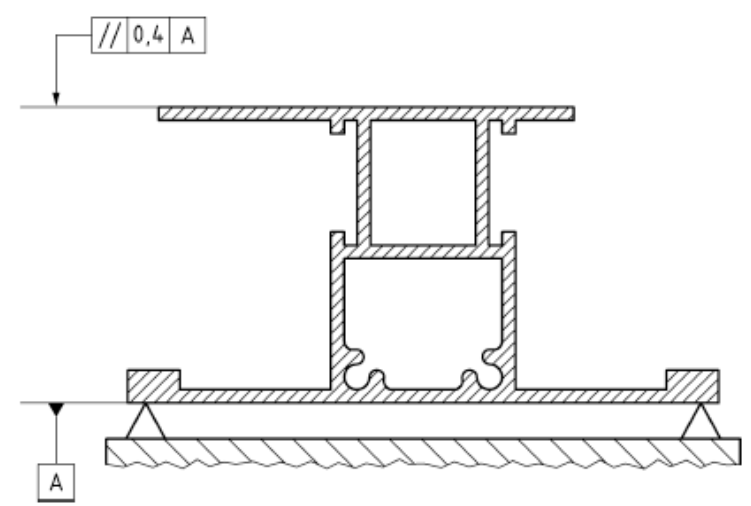

Fig. 2. Unclear indication with the caption "marking parallelism for convex and concave surfaces" [3]

Figure 3 presented below is an extract from the "Contour" clause [3]. The term closest to "theoretical exact line" may be interpreted as a synonym for "theoretically exact feature (TEF)" and can be found in [5] along with proper definition. It can be applicable for this example when combining with other fundamental GPS rules (see Section 3). It is astonishing that the EN 12020-2 standard applies this definition only for cross-sections of curved surfaces because it could be also applied for all sections of planar surfaces. Again, the term used in [3] is not in line with terms well defined in [4] although ISO 1101 is listed in EN 12020-2 as the normative reference.

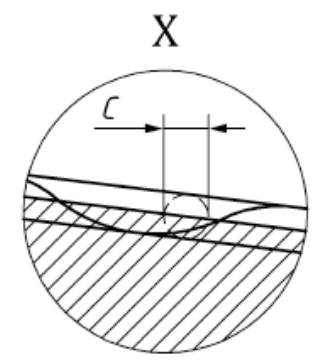

Fig. 3. Definition of contour tolerance given in [3] is similar to the definition of tolerance zone for line profile [5] 
The terms "convexity - concavity" (Fig. 4) refer to form deviations and are closest to "straightness" [4] however, the standard does not indicate this tolerance and it is not clear if tolerance values given in Table 5 [3] apply to a cross-section or to the whole profile length (in such cases "flatness" shall be applicable). The head in Table 5 related to drawings given in [3] (Fig. 4) has the caption "Width" and in the keys for the figures given in [3], dimensions indicated as $W$ are captioned as width. On the other hand, the term "straightness" is already used in EN 12020-2 in subclause 4.2, so it seems that "convexity - concavity" is not a well defined 2D requirement.
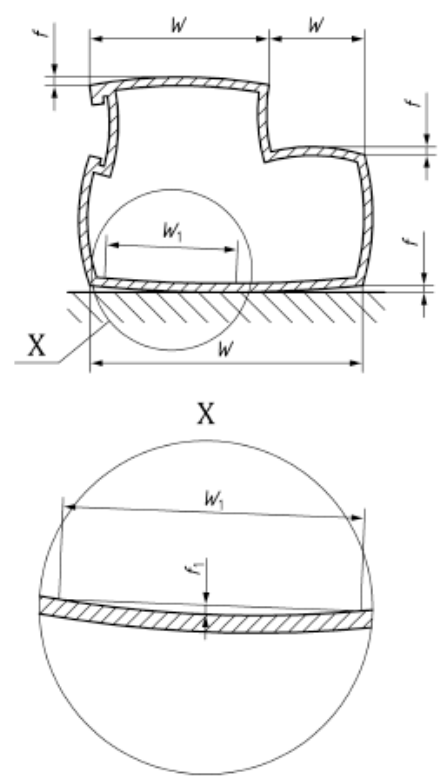
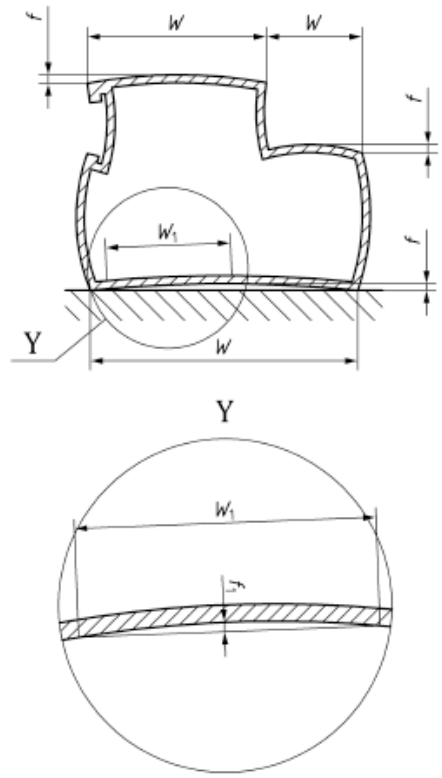

Fig. 4. The concept of convexity - concavity measurement [3] is linked with verification of straightness tolerance on the restricted area [4]

Definition of the term "Twist" (Fig. 5) along with the definition of differing positioning of the profile and discontinuous twist over the profile length provides for multiple allowable interpretations. Authors of the standard admit that the term might be a difficult interpretation. Only employment of a datum system [11] with orientation and location tolerances for extruded profile features would clearly define datum features and make the interpretation unambiguous.

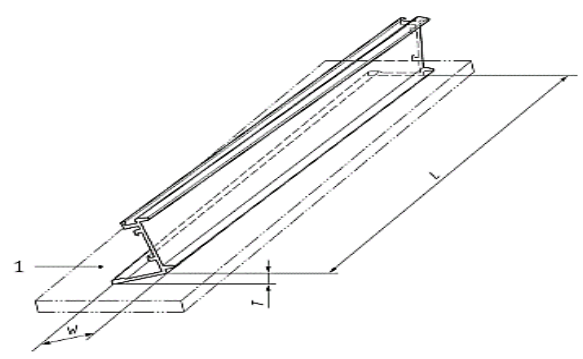

Fig. 5. Unfortunately, the sketch showing measurement of twist provided in [3] gives obscure measurement set-up on most of the actual parts 
The term "Angularity" presented in EN 12020-2 (Fig. 6) has nothing in common with the "Angularity" definition given in [4]. Taking into consideration the ISO GPS symbols and rules for their interpretations, this is a position tolerance of a point in the horizontal direction. Therefore, combinations of $+/-$ tolerancing to a dimension other than linear with the angularity definition provided in EN 12020-2 leads to unclear geometry and results in an unambiguous assessment in most cases of actual parts with deviations. A similar situation occurs when general tolerances [14] are applied to a workpiece definition. For this reason, the Technical Committee ISO TC/213 prepared the new standard [15] which can be used to simplify tolerancing and eliminate ambiguities for less functionally important geometrical features and [14] is already withdrawn.

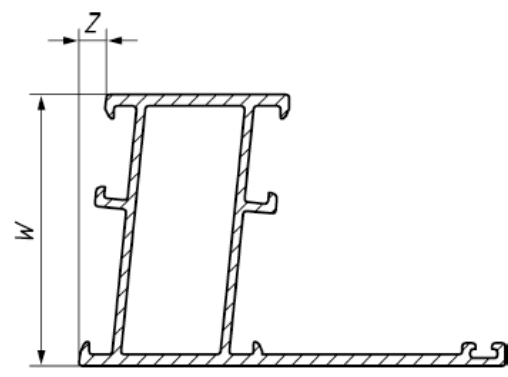

Fig. 6. The concept of angularity [3] is not totally in line with the terms given in [4]

Furthermore, the title of clause 4 (Tolerances of form) [3] is not in line with the clause content. Actually, clause 4 refers to form and orientation tolerances, which emphasizes a lack of clear structure of the standard and is a glaring error, as form cannot control an orientation specification [4]. Besides that, the standard contains more recommendations that directly lead to unclear situations such as the usage of gauges without a formal call-out of an unrelated or related mating envelope [16]. Lack of standardization for the gauges description (defined in [17] which also belongs to the GPS matrix) may lead to significant differences occurring during tool validation at customer and supplier locations.

The standard recommends multiple times $(11 \mathrm{x})$ agreements between supplier and purchasers instead of unambiguous requirement definitions. It is hardly possible to describe all interpretation combinations and attach them to a contract that refers to a drawing. The ISO GPS system provides tools where requirements based on geometrical specifications have no, or very low, specification ambiguity. Such a high number of agreement recommendations calls into question the usefulness of EN 12020-2. Therefore, in the next section some significant improvements for terms included in EN 12020-2 and the wider incorporation of the ISO GPS system terms, definitions, and rules were proposed to describe geometrical requirements for extruded profiles.

\section{NEW WAYS FOR SPECIFICATION OF EXTRUDED PROFILE GEOMETRY}

The simplified version of the drawing used in [3] (Fig. 7) shows an example of the use of $+/-$ tolerances. The values are taken from Table 1 [3] and are used in other examples in 
this section for dimensions other than size. Such dimensioning is ambiguous and results in high specification ambiguity. This specification ambiguity means that more than one interpretation of a requirement is possible and can be used to prove conformance with the requirement. This is due to the geometrical deviations of the real workpiece.

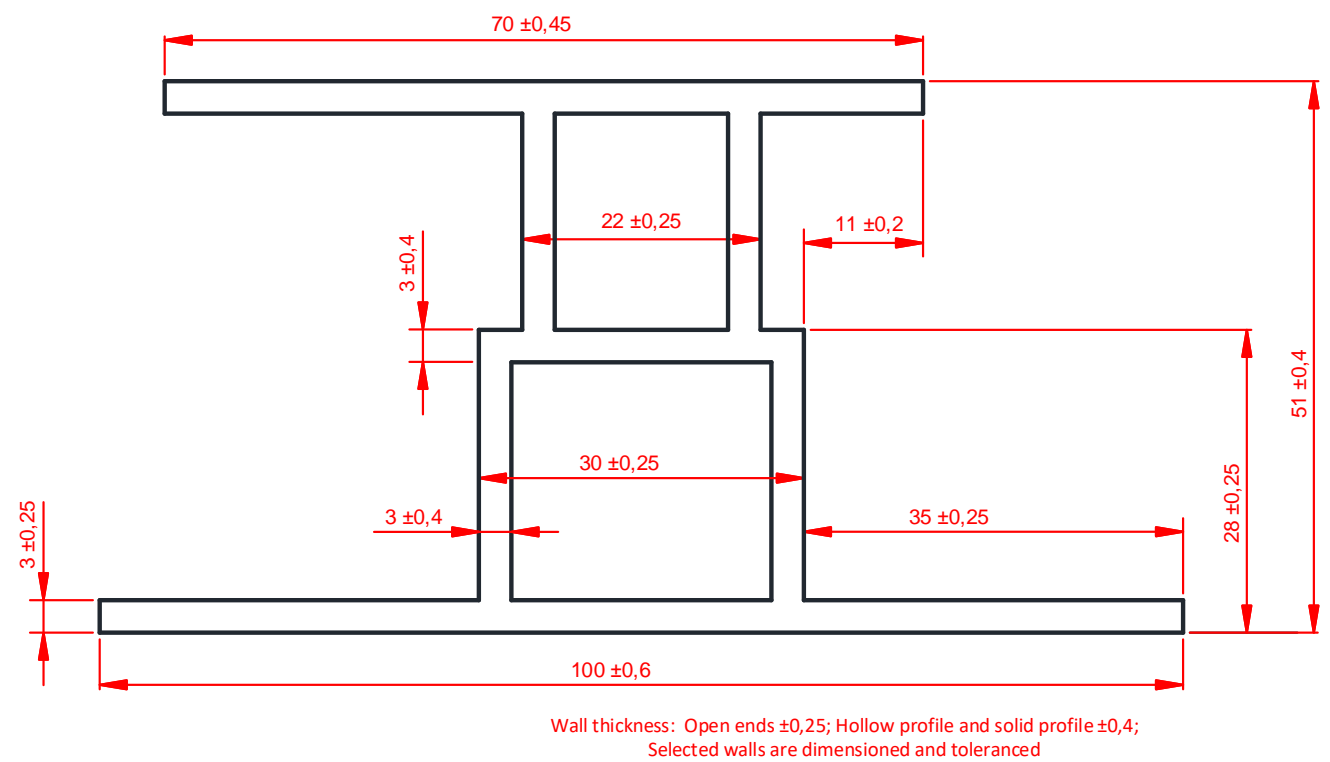

Fig. 7. Plus/minus tolerancing for all dimensions based on recommendations given in [3]

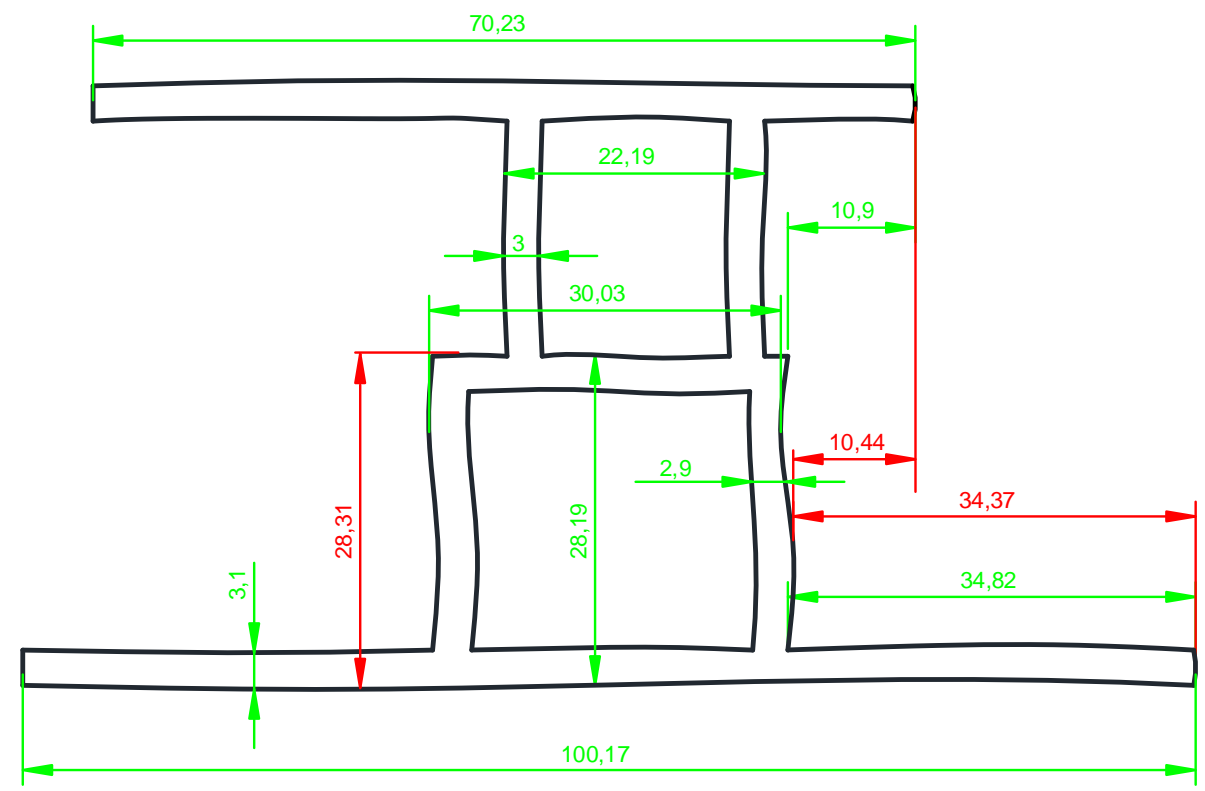

Fig. 8. Actual imperfect geometry of a profile cross-section. Dimensions indicated in red are out of specification and dimensions indicated in green are within specification

An example of an actual profile with geometrical deviations is presented in Fig. 8 . The cross-section of the profile deviates from the nominal shape, but the majority of the dimensional requirements are met. Lack of a proper datum system results in two different possible results (within and not-within limits) for dimensions $28,11,35$, which are caused by 
form deviations of surfaces and lack of a proper datum system. The most visible difference is the location of an upper hollow space with respect to a lower hollow space caused by lack of position tolerance with respect to the selected datum system.

Fig. 9 shows a solution with a proper workpiece datum system used for orientation and location tolerances as well as for form tolerances. It results in no, or very low, specification ambiguity. Tolerances given in Table 1 [3] apply only to features of size; tolerances on form and orientation are based on definitions given in [4] and their values were based on co-author experiences. Three levels of requirements are included in the workpiece definition:

- tolerances applicable for any cross-section (indicated by modifier ACS) with respect to the datum $\mathrm{A}$ or datum system $|\mathrm{A}| \mathrm{B} \mathbb{M} \mid$,

- tolerances applicable for the restricted area $-300 \mathrm{~mm}$ profile length with respect to the datum $\mathrm{C}$ or the datum system $|\mathrm{C}| \mathrm{E}$ (M) $\mid$

- tolerances applicable by default on full profile length with respect to the datum D or the datum system $|\mathrm{D}| \mathrm{F}(\mathrm{M}) \mid$.

In all cases for all wall thicknesses, as an additional constraint, the values $3 \pm 0.4$ or \pm 0.25 apply as dimensional tolerances depending on wall function (Table 2 [3]).

It is worth mentioning that although in [3], the cross-section is repeatedly referred to, there not any provisions how such cross-section shall be extracted on the actual extruded profile. Therefore, we propose to establish the cutting plane that gives the cross-section of the lowest possible circumscribed diameter. Such rule shall be given in the new edition of [2] and [3].

The maximum material requirement (MMR) is specified for perpendicularity, position and symmetry tolerances for features of size that can be distinguished in the extruded profile (Fig. 9) because the intended function is assembly with clearance with a respective mating part. The assembly of parts depends on the combined effect of the size and geometrical deviation of the toleranced features. Due to the application of the MMR the thin aluminium sheet or the $300 \mathrm{~mm}$ long slider can move along the total length of the extruded profile. The functional tolerance is not distributed on size tolerance and geometrical tolerances but is specified for both. The geometrical deviation may be larger if a feature of size is more away from its maximum material size. Application of the MMR is beneficial for the production costs and enables quick functional verification of the extruded profile with the usage of the hard gauge. The MMR specification for the total length of the extruded profile is a consequence of MMR application for cross-section and restricted length. It is functionally unlikely that two profiles will be assembled on their total length but MMR for total length limits geometrical deviations of the extruded profile in a smart way.

For any cross-section, the integral, nominally straight line (with max. straightness deviation $0.2 \mathrm{~mm}$ ), which is not a feature of size is used to establish the datum A (Fig. 9). In this cross-section due to the specification element (Any Cross-section) collection of two nominally parallel lines, which is a feature of size, is used to establish the datum B with orientation constraints (perpendicularity) between the datums and by considering the size variable. The extracted feature of the datum feature B: shall not violate the maximum material virtual condition, MMVC [16], which has the maximum material virtual size MMVS = $30.25 \mathrm{~mm}$ and in all places it shall have a two-point local size larger than LMS $=29.75 \mathrm{~mm}$ and smaller than MMS $=30.25 \mathrm{~mm}$. The extracted feature of the bottom shelf sides (the 
toleranced feature) due to symmetry tolerance $0.5 \mathbb{M}$ shall not violate the MMVC, which has MMVS $=101.1 \mathrm{~mm}$ and in all places it shall have a local size larger than LMS $=99.4 \mathrm{~mm}$ and smaller than MMS $=100.6 \mathrm{~mm}$.

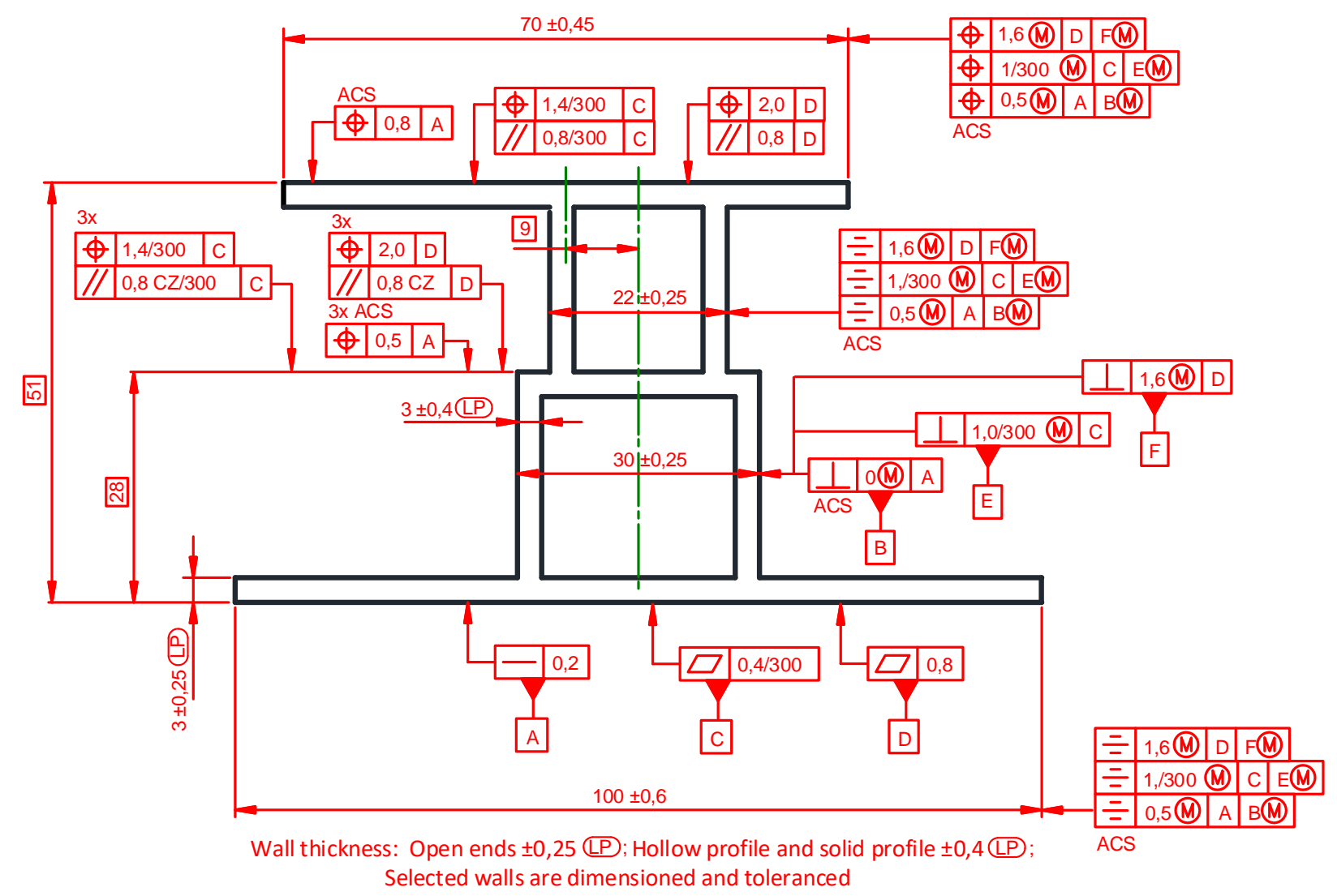

Fig. 9. Extruded profile depicting geometrical features with restricted form, orientation and location (1st proposal of geometrical tolerancing). The straightness deviation shall be determined in the cross-section of the lowest possible circumscribed diameter

The location of MMVC is at the theoretically exact dimension TED $=0 \mathrm{~mm}$ from the symmetry line of the MMVC of the secondary datum feature B. The extracted feature of the upper hollow (space toleranced feature) shall not violate the MMVC [16], which has the size $22.75 \mathrm{~mm}$ and in all places it shall have a local size larger than LMS $=21.75 \mathrm{~mm}$ and smaller than MMS $=22.25 \mathrm{~mm}$. The location of MMVC is at the theoretically exact dimension $0 \mathrm{~mm}$ from the symmetry line of the MMVC of the secondary datum feature B. The extracted feature of the upper shelf sides (the toleranced feature of size) shall not violate the MMVC [16], which has MMVS $=70.95 \mathrm{~mm}$ and in all places it shall have a local size larger than LMS = $69.55 \mathrm{~mm}$ and smaller than MMS $=70.45 \mathrm{~mm}$. The location of the MMVC of the upper hollow space is at theoretically exact distance TED $=9 \mathrm{~mm}$ from the symmetry plane of the MMVC of the datum feature B. The tolerance zone of the lower line segment of the upper hollow space (+ two external side-line segments) defined by the specification is limited by a pair of parallel straight lines a distance of $0,5 \mathrm{~mm}$ apart, symmetrically disposed about their theoretically exact position and fixed by a theoretically exact dimension TED $=28 \mathrm{~mm}$ with respect to the datum $\mathrm{A}$. The tolerance zone of the upper line of the upper shelf defined by 
the specification is limited by a pair of parallel straight lines a distance of $0.8 \mathrm{~mm}$ apart, symmetrically disposed about the theoretically exact position fixed by the theoretically exact dimension of $51 \mathrm{~mm}$ with respect to the datum A.

The new method of tolerancing for any cross-section of the aluminium alloy-extruded profile has been proposed above. The geometry of each cross-section is important, but it is not enough from a functional point of view - extruded profiles imply that a profile is mating along its length. So further geometrical requirements shall be specified for restricted areas of a profile geometrical features and finally for total extend of a profile geometrical features.

The restricted $300 \mathrm{~mm}$ extruded profile length of the integral, nominally planar surface which is not a feature of size is used to establish the datum $\mathrm{C}$. The flatness tolerance $0.4 \mathrm{~mm}$ at any restricted length $300 \mathrm{~mm}$ and by default full width $100 \mathrm{~mm}$ of the bottom shelf is specified for this restricted datum. The collection of two nominally parallel planar surfaces (over a restricted area $300 \mathrm{~mm}$ length), which is a feature of size, is used to establish the datum $\mathrm{E}$ with orientation constraints (perpendicularity) between the secondary and primary datums and by considering the size variable. The extracted feature of the datum feature E shall not violate the maximum material virtual condition, MMVC, which has the size MMVS $=31.25 \mathrm{~mm}$ and in all places it shall have a two-point size larger than LMS $=29.75 \mathrm{~mm}$ and smaller than MMS $=30.25 \mathrm{~mm}$. The extracted feature of the bottom shelf sides (toleranced feature) shall not violate the MMVC, which has the size $101.6 \mathrm{~mm}$ and in all places it shall have a local size larger than $\mathrm{LMS}=99.4 \mathrm{~mm}$ and smaller than $\mathrm{MMS}=100.6$ $\mathrm{mm}$. The location of the MMVC of the bottom shelf is at the theoretically exact dimension $\mathrm{TED}=0 \mathrm{~mm}$ from the symmetry plane of the MMVC of the datum feature E. The extracted feature of the upper hollow space (toleranced feature) shall not violate the MMVC [16], which has the size $23.25 \mathrm{~mm}$ and in all places it shall have the two-point size larger than LMS = $21.75 \mathrm{~mm}$ and smaller than MMS $=22.25 \mathrm{~mm}$. The location of the MMVC is at the theoretically exact dimension TED $=0 \mathrm{~mm}$ from the symmetry plane of the MMVC of the datum feature E. The extracted feature of the upper shelf sides shall not violate the MMVC, which has the size $71.45 \mathrm{~mm}$ and in all places it shall have a local size larger than LMS = $69.55 \mathrm{~mm}$ and smaller than MMS $=70.45 \mathrm{~mm}$. The location of MMVC is at the theoretically exact dimension $9 \mathrm{~mm}$ from the symmetry plane of the MMVC of the datum feature E. The tolerance zone of the restricted lower surface of the upper hollow space $(+$ two sides of restricted surfaces) defined by the specification is limited by a pair of parallel planes a distance of $1.4 \mathrm{~mm}$ apart, symmetrically disposed about the theoretically exact position and fixed by the theoretically exact dimension of $28 \mathrm{~mm}$ with respect to datum $\mathrm{C}$. The tolerance zone of the upper restricted surface of the upper shelf defined by the specification is limited by a pair of two parallel planes a distance of $1.4 \mathrm{~mm}$ apart, symmetrically disposed about the theoretically exact position and fixed by the theoretically exact dimension of $51 \mathrm{~mm}$ with respect to the datum $\mathrm{C}$.

For all tolerances listed above the restricted area means the rectangle of $300 \mathrm{~mm}$ length specified after the tolerance value and separated from it by an oblique stroke and width by default defined by nominal dimensions of an extruded profile cross-section.

The full profile length of the integral nominally planar surface which is not a feature of size is used to establish the datum D. The flatness tolerance of the full profile length is equal to $0.8 \mathrm{~mm}$. The collection of two nominally parallel planar surfaces, which is a feature 
of size is used to establish a datum F with orientation constraints (perpendicularity) with respect to primary datum and by considering the size variable. The extracted feature of the datum feature shall not violate the maximum material virtual condition, MMVC, which has the size MMVS $=31.85 \mathrm{~mm}$ and in all places it shall have a local size larger than LMS = $29.75 \mathrm{~mm}$ and smaller than MMS $=30.25 \mathrm{~mm}$. The extracted feature of the bottom shelf sides shall not violate the MMVC [16], which has the size $102.2 \mathrm{~mm}$ and in all places it shall have a two-point size larger than LMS $=99.4 \mathrm{~mm}$ and smaller than $\mathrm{MMS}=100.6 \mathrm{~mm}$. The location of the MMVC is at the theoretically exact dimension $0 \mathrm{~mm}$ from the symmetry plane of the MMVC of the datum feature F. The extracted feature of the upper hollow space shall not violate the MMVC, which has the size $23.85 \mathrm{~mm}$ and in all places it shall have everywhere a local size larger than LMS $=21.75 \mathrm{~mm}$ and smaller than MMS $=22.25 \mathrm{~mm}$. The location of the MMVC is at the theoretically exact dimension $0 \mathrm{~mm}$ from the symmetry of the MMVC of the datum feature F. The extracted feature of the upper shelf shall not violate the MMVC, which has the size $72.05 \mathrm{~mm}$ and in all places it shall have a local size larger than LMS = $69.55 \mathrm{~mm}$ and smaller than MMS $=70.45 \mathrm{~mm}$. The location of MMVC is at the theoretically exact dimension $9 \mathrm{~mm}$ from the symmetry plane of the MMVC of the datum feature F. The tolerance zone of the lower surface of the upper hollow space (+ two side surfaces) defined by the specification is limited by a pair of parallel planes a distance of $2 \mathrm{~mm}$ apart, symmetrically disposed about the theoretically exact position and fixed by the theoretically exact dimension $28 \mathrm{~mm}$ with respect to the datum $\mathrm{D}$. The tolerance zone of the upper surface of the upper shelf defined by the specification is limited by a pair of parallel planes a distance of $2 \mathrm{~mm}$ apart, symmetrically disposed about the theoretically exact position and fixed by the theoretically exact dimension $51 \mathrm{~mm}$ with respect to the datum D.

Fig. 10 shows a geometrical specification of integral features, using the line profile and surface profile characteristic symbols for a set of features (straight lines and planes). "All around" indication apply to a united feature (UF). The specification does not reference datums, but due to indication of tolerance feature identifiers UF and "all around" it controls dimensions, form as well as orientation and location among all features of an extruded profile. The applied tolerances specify boundaries with respect to the theoretically exact profile within which all points of an extruded profile shall lie.

Applied tolerance values are based on Table 6 [3] and co-author experiences. Three levels of requirements are included in the workpiece definition:

- tolerances applicable for any cross-section; we propose to establish the cutting plane that gives a cross-section of the lowest possible circumscribed diameter,

- tolerances applicable for the restricted area - $300 \mathrm{~mm}$ profile length,

- tolerances applicable on full profile length.

In all cases, for all wall thicknesses, as a further constraint, the value $3 \pm 0.4$ or \pm 0.25 apply as dimensional tolerances depending on wall function (Table 2 [3]), independently from $\mathrm{TED}=3 \mathrm{~mm}$ which defines theoretical exact feature. This approach is used in [18]. Lack of two-point local size call-out in any cross-section would end up in a possible $2 \mathrm{~mm}$ wall thicknesses due to line profile tolerance with the tolerance value $1 \mathrm{~mm}$.

Theoretically exact feature is defined with theoretically exact dimensions. Because the "all around" symbol and the UF (united feature) modifier are used, the specification applies to a united feature built from the features that make up the periphery of the workpiece 
when seen in a cutting plane which is determined in each cross-section as the plane containing the circle of the lowest possible circumscribed diameter. The toleranced features are a line (for any cross-section) or a surface (two other levels of requirements). The tolerance zone is limited by two equidistant lines (respectively surfaces) enveloping circles (respectively spheres) with a diameter equal to the tolerance value, the centres of which are situated on the theoretically exact feature (TEF). Because the OZ (offset zone) modifier is used, the tolerance zone is allowed an unspecified, but constant, offset from TEF. This way the offset tolerance zone controls the form of each toleranced feature within the larger, tolerance zone that controls dimensions and relative locations of all geometrical features of an extruded profile.

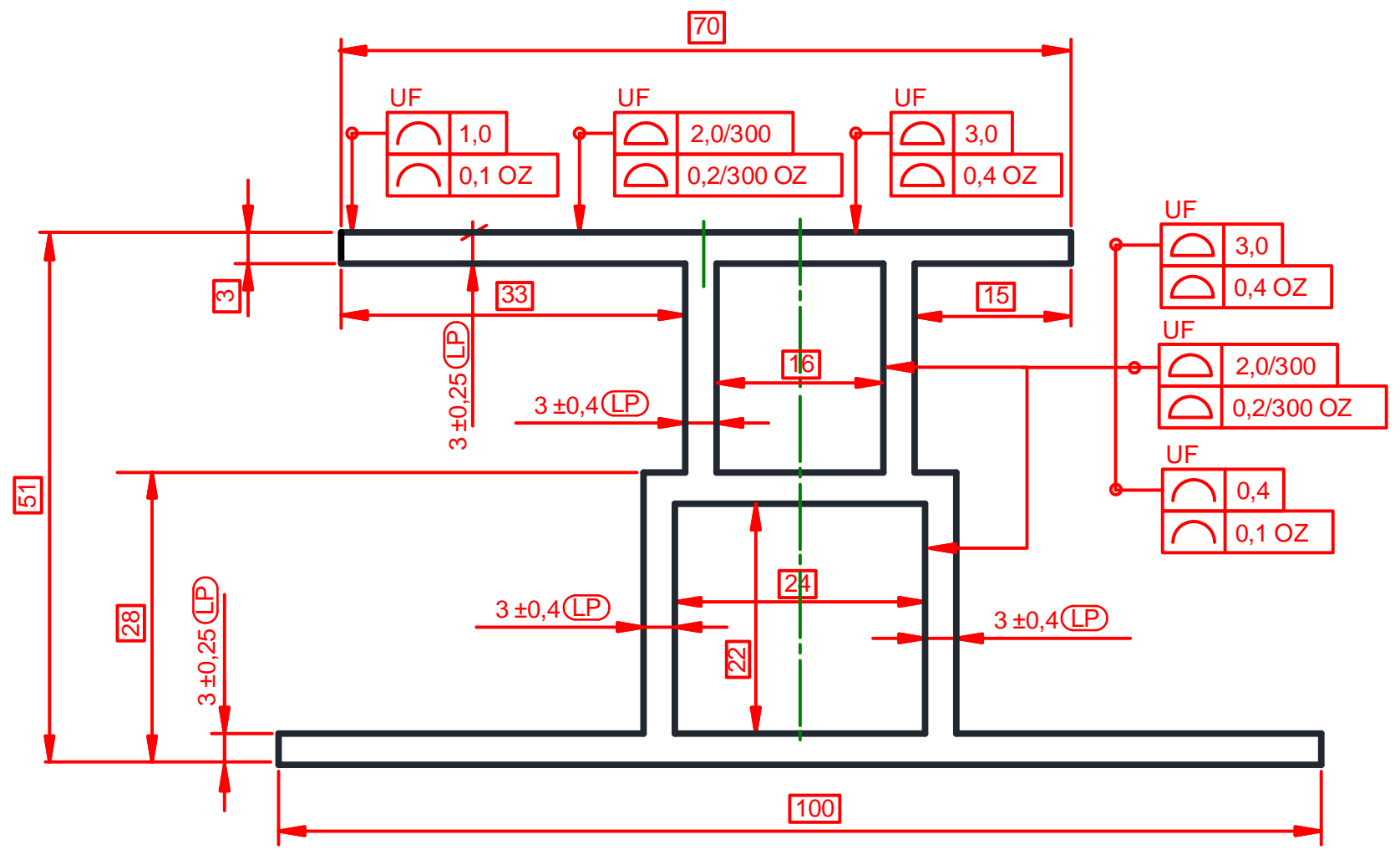

Wall thickness: Open ends $\pm 0,25$ (LP); Hollow profile and solid profile $\pm 0,4$ (LP); Selected walls are dimensioned and toleranced

Fig. 10. Extruded profile geometrical features with restricted form, orientation and location (2nd proposal of geometrical tolerancing). The line profile deviations shall be determined in the cross-section of the lowest possible circumscribed diameter

\section{SUMMARY}

The discussed examples of ambiguous definitions show there is no doubt that the current version of [3] leaves too much vagueness and laxity in the definitions in terms of requirement verification. Rules, terms, and definitions included in the ISO GPS system can be easily used to describe an extruded profile geometry in multiple ways depending on design intent. To 
avoid insufficiencies of non-GPS standards used in product definition in different manufacturing processes and to meet industry expectations, ISO published a few standards which are part of the GPS matrix, e.g., for castings [18]). However, no actions have been taken concerning the aluminium extrusion industry. Therefore, a review of [3] is worthwhile.

Only two proposals of the geometrical tolerances application for a consistent, unique definition of an extruded profile geometry are given in this paper. A few other alternatives seem to be possible. Due to industry expectations, the authors intend to explore the application of other geometrical tolerances.

\section{REFERENCES}

[1] https://autostoresystem.com/.

[2] EN 755-9:2016 Aluminium and aluminium alloys - Extruded rod/bar, tube and profiles - Part 9: Profiles, tolerances on dimensions and form.

[3] EN 12020-2:2016 Aluminium and aluminium alloys - Extruded precision profiles in alloys EN AW-6060 and EN AW-6063 - Part 2: Tolerances dimensions and form.

[4] ISO 1101:2017 Geometrical product specifications (GPS) - Geometrical tolerancing - Tolerances of form, orientation, location and run-out.

[5] ISO 1660:2017 Geometrical product specifications (GPS) - Geometrical tolerancing - Profile tolerancing.

[6] OEZDEN R., POPA M.S., DANHO S., 20119, Analysis and Impact of Different Ambiguities in Industrial Technical Product Drawings, IOP Conf. Series, Materials Science and Engineering, 564, 012047.

[7] ISO 14405-1:2016 Geometrical product specifications (GPS) - Dimensional tolerancing - Part 1: Linear sizes.

[8] ISO 14405-3:2016 Geometrical product specifications (GPS) - Dimensional tolerancing - Part 3: Angular sizes.

[9] ISO 14405-2:2018 Geometrical product specifications (GPS) - Dimensional tolerancing - Part 2: Dimensions other than linear or angular sizes.

[10] ISO 17450-3:2016 Geometrical product specifications (GPS) - General concepts - Part 3: Tolerance features.

[11] ISO 5459:2011 Geometrical product specifications (GPS) - Geometrical tolerancing - Datums and datum systems

[12] HENZOLD G., 2020, Geometrical Dimensioning \& Tolerancing for Design, Manufacturing and Inspection, A handbook for Geometrical Product Specification using ISO and ASME standards, Butterworth-Heinemann.

[13] TORNINCASA S., 2020, Technical Drawing for Product Design, Mastering ISO GPS and ASME GD\&T, Springer.

[14] ISO 22081:2021 Geometrical product specifications (GPS) - Geometrical tolerancing - General geometrical specifications and general size specifications.

[15] ISO 2768-2:1989 General tolerances - Part 2: Geometrical tolerances for features without individual tolerance indications.

[16] ISO 2692:2014 Geometrical product specifications (GPS) - Geometrical tolerancing - Maximum material requirement (MMR), least material requirement (LMR) and reciprocity requirement (RPR).

[17] ISO 1938-1:2015 Geometrical product specifications (GPS) - Dimensional measuring equipment - Part 1: Plain limit gauges of linear size.

[18] ISO 8062-4:2017 Geometrical product specifications (GPS) - Dimensional and geometrical tolerances for moulded parts - Part 4: General tolerances for castings using profile tolerancing in a general datum system. 